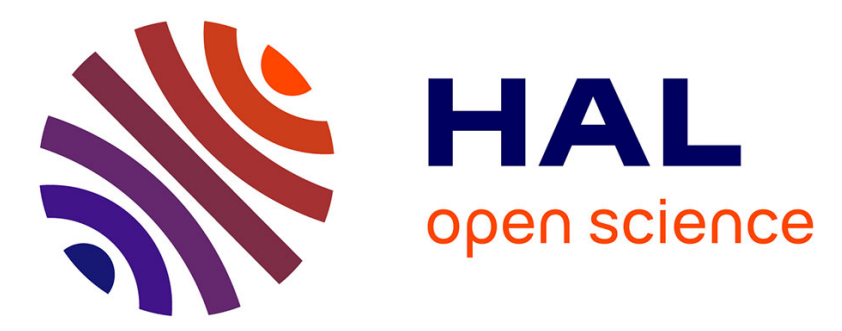

\title{
On the relationship between C-C and C-D order parameters and its use for studying the conformation of lipid acyl chains in biomembranes
}

\author{
Jean-Paul Douliez, A. Ferrarini, E.J. Dufourc
}

\section{- To cite this version:}

Jean-Paul Douliez, A. Ferrarini, E.J. Dufourc. On the relationship between C-C and C-D order parameters and its use for studying the conformation of lipid acyl chains in biomembranes. Journal of Chemical Physics, 1998, 109 (6), pp.2513-2518. hal-02696454

\section{HAL Id: hal-02696454 \\ https://hal.inrae.fr/hal-02696454}

Submitted on 1 Jun 2020

HAL is a multi-disciplinary open access archive for the deposit and dissemination of scientific research documents, whether they are published or not. The documents may come from teaching and research institutions in France or abroad, or from public or private research centers.
L'archive ouverte pluridisciplinaire HAL, est destinée au dépôt et à la diffusion de documents scientifiques de niveau recherche, publiés ou non, émanant des établissements d'enseignement et de recherche français ou étrangers, des laboratoires publics ou privés.

$$
\text { Copyright }
$$




\title{
On the relationship between $C-C$ and $C-D$ order parameters and its use for studying the conformation of lipid acyl chains in biomembranes
}

\author{
J.-P. Douliez ${ }^{\text {a) }}$ \\ A. Ferrarini \\ Department of Physical Chemistry. University of Padua, 35131 Padova, Italy \\ E.-J. Dufourc \\ Centre de Recherche Paul Pascal, CNRS, av. A. Schweitzer, 33600 Pessac, France
}

Laboratoire de Biochimie et Technologie des Protéines, INRA, av. de la Géraudière 44316 Nantes, France

(Received 24 February 1998; accepted 5 May 1998)

\begin{abstract}
We have used the order tensor method to establish relationships between carbon-carbon and carbon-deuterium order parameters ( $S^{\mathrm{CC}}$ and $S^{\mathrm{CD}}$, respectively) in saturated and unsaturated lipid acyl chains in biomembranes. We thus confirm the existence of a recursion relation between these order parameters $\left(-2 S_{k}^{\mathrm{CD}}=S_{k}^{\mathrm{CC}}+S_{k+1}^{\mathrm{CC}}\right)$ as was previously shown using Wigner matrices formalism [Douliez, Léonard, and Dufourc, Biophys. J. 68, 1727 (1995)]. This leads to the determination of $S_{k}^{C C}$ as a function of the position $k$ along the chain, having experimentally measured the corresponding $S_{k}^{C D}$. Making use of a mean field approach for computing the energy of chain conformers in the frame of the rotational isomeric model (RIS) we have also calculated $S^{\mathrm{CC}}$ and $S^{\mathrm{CD}}$ order profile for the $s n-1$ chain of dipalmitoylphosphatidylcholine. A very good agreement is obtained between computational and predicted $S_{k}^{\mathrm{CC}}$ values, reinforcing the validity of the recursion relation. A marked odd-even effect is evidenced on the $S^{C C}$ order profile for positions near the polar head, as was previously experimentally reported for dimyristoylphosphatidylcholine. We propose that this effect reveals an average bent orientation of the beginning of the chain with respect to the normal of the bilayer. Finally, it is shown that our formalism can be applied to some extent to cis or trans unsaturated lipid. (c) 1998 American Institute of Physics. [S0021-9606(98)51530-9]
\end{abstract}

\section{INTRODUCTION}

Conformation and dynamics of lipid acyl chains are of strong interest for understanding the functional properties of membranes. For such studies, a great deal of interest has been devoted to computational methods. Information about static and dynamic structure, through the calculation of order parameters or correlation functions may be provided from Monte Carlo, ${ }^{1-3}$ master equation, ${ }^{4}$ maximum entropy, 5,6 Brownian dynamics ${ }^{7}$ and molecular dynamics simulations. ${ }^{8-11}$ As an example, order parameters calculated from these methods can be compared to experimental data from NMR (Refs. 12, 13) or Raman scattering ${ }^{14}$ whereas correlation functions may be connected to $T_{1}$ relaxation experiments. ${ }^{15.16}$ The relative ease in deuterating lipid acyl chains and the use of solid state NMR has led to the appearance of C-D order parameter profiles. They have been widely studied theoretically during the last two decades ${ }^{12,15,17-20}$ and provide information on acyl chain length or number of gauche defects ${ }^{20,21}$ as well as on bilayer elastic constants. ${ }^{22-25}$ Recent progress in pulse sequence methodology has allowed measurement of sign and intensity of $\mathrm{C}-\mathrm{H}$ dipolar couplings, which yields similar orientational information. ${ }^{26-28}$ Double quantum and separated local field experiments provide valuable determination of $\mathrm{C}-\mathrm{H}$ and $\mathrm{C}-\mathrm{C}$ dipolar couplings without requiring isotopic labeling. ${ }^{19,29-31}$

\footnotetext{
atAuthor to whom correspondence should be addressed.
}

While $\mathrm{C}-\mathrm{H}$ dipolar coupling and $\mathrm{d}$ adrupolar interaction bring the same information, e.g., $S_{k} \mathrm{br} S_{k}^{\mathrm{CD}}$, C-C couplings provide additional details on local segmental motion. We have previously developed a formalism allowing calculation of $S_{k}^{C C}$ from experimental $S_{k}^{\mathrm{CD}} \cdot{ }^{20}$ It links two adjacent C-C order parameters of a methylene unit, $k$, to the corresponding $S_{k}^{C D}$ (Fig. 1) and yields the rectirsion relation:

$$
-2 S_{k}^{\mathrm{CD}}=S_{k}^{\mathrm{CC}}+S_{k+1}^{\mathrm{CC}} \text {. }
$$

This equation was initially derived by performing coordinate transformations from the C-C or C-D bonds of a lipid chain to the bilayer normal, n. For ease of calculation, Wigner matrices were used and a complete decomposition of motions occurring at the NMR time scale was performed. As a consequence, it was not clearly demonstrated whether this recursion relation depends or not on the dynamics occurring in the membrane. In the same way, the terminal bond order parameter, $S_{n}^{C C}$, was determined by a simple coordinate transformation from the $\mathrm{C}_{n}-\mathrm{D}$ bond of the methyl terminus to the $\mathrm{C}_{n-1}-\mathrm{C}_{n}$ bond (which is also the rotation axis of the methyl group). Subsequently, the recursion relation was used to calculate all the C-C bond order parameters along the chain having started from the methyl-end. Let us note that Eq. (1) stands only for the case of saturated chains and to our knowledge, no similar relation has been yet established in the case of a double bond. It should be mentioned that, as a consequence of Eq. (1), $S_{k}^{\mathrm{CD}}$ and $S_{k}^{\mathrm{CC}}$ bring the same structural and dynamical information. However, it is more conve-

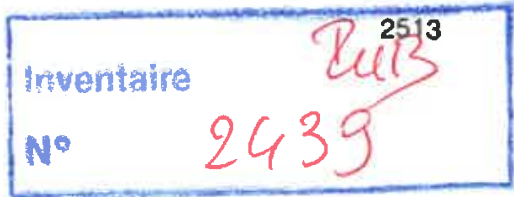




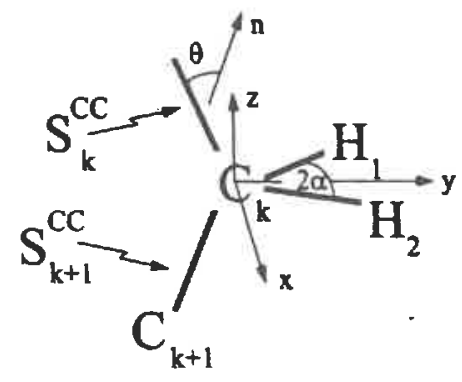

FIG. 1. Schematic representation of a methylene group in a lipid acyl chain with the axes systems needed for the description of order parameters. $\mathbf{n}$ is the normal to the bilayer and the $x . y, z$ frame is defined as in van det Ploeg and Berendsen (Ref. 9). $S_{k}^{\mathrm{CC}}$ and $S_{k+1}^{\mathrm{CC}}$ is respectively the onder parameter of the $\mathrm{C}_{k-1}-\mathrm{C}_{k}$ and $\mathrm{C}_{k}-\mathrm{C}_{k+1}$ bond. $2 a$ is the angle between the $\mathrm{CH}_{1}$ and $\mathrm{CH}_{2}$ vectors.

nient to use the $S^{\mathrm{CC}}$ formalism as a basis to calculate physical parameters such as acyl chain length or number of gauche defects. Moreover, the $S^{\mathrm{cC}}$ order profile may reveal an oddeven effect that cannot be observed for $S^{\mathrm{CD}}$ measured from perdeuterated chains. The amplitude of such an effect has been found to increase with temperature for $s n-2$ chains of several lipids whereas it remains constant for $s n-1$ chains. ${ }^{20.21}$ The validity of the formalism has been tested experimentally from ${ }^{13} \mathrm{C}$ - and ${ }^{2} \mathrm{H}-\mathrm{NMR}$ experiments. Palmitic acid deuterated on the whole chain and labeled with ${ }^{13} \mathrm{C}$ on the $C_{1}$ and $C_{2}$ positions were embedded in a liquid crystal. $S_{k}^{\mathrm{CD}}$ were then measured allowing calculation of $S_{k}^{\mathrm{CC}}$ (as described above) and especially $S_{2}^{\mathrm{cC}}$, the order parameter of the $\mathrm{C}_{1}-\mathrm{C}_{2}$ bond. This former order parameter was also experimentally determined with one and two-dimensional ${ }^{13} \mathrm{C}-\mathrm{NMR}$ by measuring the dipolar coupling between $\mathrm{C}_{1}$ and $\mathrm{C}_{2}$. Both values of $S_{2}^{\mathrm{CC}}$ determined separately as a function of temperature were found in very good agreement. ${ }^{21}$

In order to generalize the validity of our formalism, we use in this paper the order tensor concept for calculating $S_{k}^{\mathrm{CC}}$ and $S_{k}^{\mathrm{CD}}$ order parameters. This approach has a strong advantage since it does not require any decomposition of motions occurring in the system. It also leads to the recursion relation, Eq. (1), demonstrating that the $S^{\text {CC }}$ formalism is dynamically independent and rather depends on the molecular geometry. By using a thermodynamical model to compute the configurational energy of the $s n-1$ chain of DPPC, we calculate $S_{k}^{\mathrm{CC}}$ and $S_{k}^{\mathrm{CD}} . S^{\mathrm{CC}}$ order parameter profiles exhibit an odd-even effect especially for positions near the polar head. It is proposed that such an effect is a consequence of the average bent conformation of the beginning of the chain. In a parallel way, we have extended our formalism to double bonds as in the oleoyl POPC chain. In such a case, it is not possible a priori to obtain a recursion relation. However, whatever the trans or cis configuration, it is shown how the $S^{\mathrm{CC}}$ formalism can help for the attribution of the pro- $\mathrm{R}$ and pro-S C-D order parameters of the double bond.

\section{EQUATIONS AND METHODS}

\section{A. Order tensor for saturated chains}

Order parameters are of strong interest in mean field, Maier-Saupe, theories. ${ }^{33-36}$ They are defined as an averaged ordinary Legendre polynomial of second order. In the most general case, this definition can be generalized by introducing the order parameter tensor, $\mathbf{S}$, having the following components: $:^{37.38}$

$$
S_{i j}=\left\langle 3 \cos \theta_{i} \cos \theta_{j}-\delta_{i j}\right) / 2,
$$

where $\theta_{i}$ denotes the angle between the bilayer normal and the molecular coordinates axes: $i, j=x, y, z, \delta_{i j}$ the Kronecker symbol and the angular brackets a time and space average. For methylene groups of saturated acyl chains in membranes this molecular frame is usually defined as follows: $x$ is parallel to the D-D (or H-H) vector, $y$ bisects the D-C $C_{k}-\mathrm{D}$ (or the H-C $-\mathrm{H}$ ) angle and $z$ is the normal on the plane spanned by the two $C_{k}-D\left(C_{k}-H\right)$ bond vectors (Fig. 1). This yields the well-known relation for $S^{\mathrm{CH}}$ or $S^{\mathrm{CD}}:{ }^{39}$

$$
S_{k}^{\mathrm{CD}}=S_{k}^{\mathrm{CH}}=\left(2 S_{x x}^{k}+S_{y y}^{k}\right) / 3 \text {. }
$$

For a better understanding on how to get the recursion relation, Eq. (1), it is now necessary to give more details on the way that leads to determine Eq. (3). Let us then define the $C_{k}-D_{1}$ and $C_{k}-D_{2}$ vectors as $x$ and $y$ components,

$$
\begin{aligned}
& C_{k} H_{1}=C_{k} D_{1}=\cos (\alpha) y-\sin (\alpha) \mathbf{x}, \\
& C_{k} H_{2}=C_{k} D_{2}=\cos (\alpha) y+\sin (\alpha) \mathbf{x},
\end{aligned}
$$

where $2 \alpha=109.5^{\circ}$ is the D-C $k^{-D}$ angle, with $\cos \alpha=1 / \sqrt{3}$ and $\sin \alpha=\sqrt{2 / 3}$. Let $\mathrm{n}$ be the bilayer normal and $\beta_{a}(a=1$ or 2) the angle between the $C_{k}-H_{a}$ bond and $n$ (Fig. 1),

$$
\begin{aligned}
& \cos \beta_{1}=\mathbf{n} \cdot \mathbf{C}_{k} \mathbf{H}_{1}=\mathbf{n} \cdot \mathbf{C}_{k} \mathbf{D}_{1}=\frac{1}{\sqrt{3}} \cos \theta_{y}-\frac{\sqrt{2}}{\sqrt{3}} \cos \dot{\theta}_{x}, \\
& \cos \beta_{2}=\mathbf{n} \cdot \mathbf{C}_{k} \mathbf{H}_{2}=\mathbf{n} \cdot \mathbf{C}_{k} \mathbf{D}_{2} \frac{1}{\sqrt{3}} \cos \theta_{y}+\frac{\sqrt{2}}{\sqrt{3}} \cos \theta_{x},
\end{aligned}
$$

where $\theta_{i}(i=x, y, z)$ is the angle between the axis $i$ and the normal to the bilayer. It is then easy to calculate the order parameter of the $C_{k} D_{1}$ and $C_{k} D_{2}$ bonds using $S_{k}^{\mathrm{CD}_{a}}$ $=\left\langle 3 \cos ^{2}\left(\beta_{a}\right)-1\right\rangle / 2$ which yields, with the help of Eqs. (2) and $(5)$ to

$$
\begin{aligned}
& S_{k}^{\mathrm{CD}_{1}}=\frac{2}{3} S_{x x}^{k}+\frac{1}{3} S_{y y}^{k}-\frac{2 \sqrt{2}}{2} S_{x y}^{k}, \\
& S_{k}^{\mathrm{CD}_{2}}=\frac{2}{3} S_{x x}^{k}+\frac{1}{3} S_{y y}^{k}+\frac{2 \sqrt{2}}{3} S_{x y}^{k} .
\end{aligned}
$$

Let us note that in the case where deuterons are equivalent, it follows from Eq. (6) that $S_{x y}^{k}$ is zero. Then, summation of both lines in Eq. (6) yields

$$
S_{k}^{\mathrm{CD}}+S_{k}^{\mathrm{CD}_{2}}=2 S_{k}^{\mathrm{CD}}=2\left(\frac{2}{3} S_{x x}^{k}+\frac{1}{3} S_{y y}^{k}\right)
$$

which is similar to Eq. (3).

We now take the advantage that methylene units bring the tetrahedral geometry which means that the $\mathrm{C}_{k-1}-\mathrm{C}_{k}-\mathrm{C}_{k+1}$ angle is also $2 \alpha$ (Fig. 1). It is then easy to rewrite Eqs. (4) to (6) for $S_{k}^{\mathrm{CC}}$ and $S_{k+1}^{\mathrm{CC}}$ by replacing $\mathrm{C}_{k}-\mathrm{D}_{1}$ and $C_{k}-D_{2}$ by $C_{k-1}-C_{k}$ and $C_{k}-C_{k+1}$, respectively (Fig. 1)

$$
\mathrm{C}_{k-1} \mathrm{C}_{k}=\cos (\alpha) \mathrm{y}-\sin (\alpha) \mathbf{z} \text {, }
$$




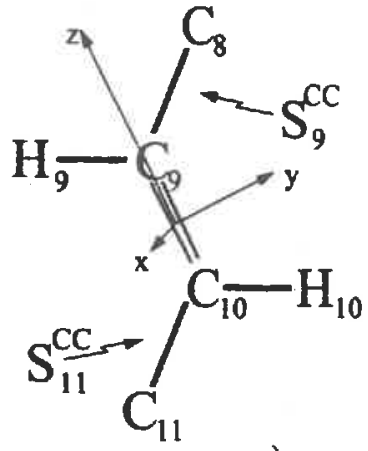

a)

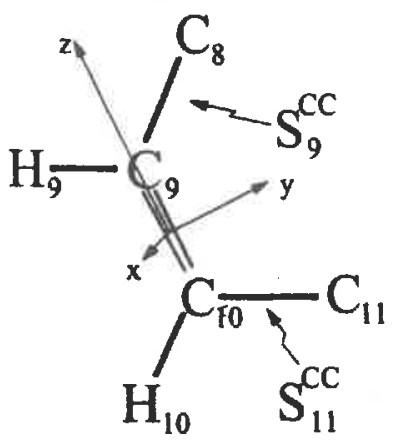

b)
FIG. 2. Schematic representation of a trans (a) and cis (b) double bond standing at positions 9 and 10 . The $x, y, z$ frame is slightly modified compared to Fig. I and is defined as in Seelig and Waespe Sarcevic (Ref. 40). Order parameters are defined as in Fig. 1, i.e., $S_{k}^{\mathrm{cC}}$ stands for the $\mathrm{C}_{k-1}-\mathrm{C}_{k}$ bond.

$$
\begin{aligned}
& \mathrm{C}_{k} \mathrm{C}_{k+1}=-\cos (\alpha) \mathrm{y}-\sin (\alpha) \mathrm{z} \\
& S_{k}^{\mathrm{CC}}=\frac{2}{3} S_{z z}+\frac{1}{3} S_{y y}-\frac{2 \sqrt{2}}{3} S_{y z} \\
& S_{k+1}^{\mathrm{CC}}=\frac{2}{3} S_{z z}+\frac{1}{3} S_{y y}+\frac{2 \sqrt{2}}{3} S_{y z}
\end{aligned}
$$

Equation (8b) expresses $S^{\mathrm{CC}}$ as a function of the $x x, y y$ order parameters and of the off-diagonal element $S_{y z}$. As mentioned in the case of equivalent deuterons, let us note that $S_{k}^{C C}=S_{k+1}^{C C}$ implies $S_{y z}$ is zero. Then, summation of both lines in Eq. (8b) gives

$$
S_{k}^{C C}+S_{k+1}^{C C}=2\left(\frac{2}{3} S_{z z}+\frac{1}{3} S_{y y}\right) \text {. }
$$

Because the trace of the order tensor is zero, the combination of Eqs. (3) and (9) leads to the recursion relationship between $S^{\mathrm{CC}}$ and $S^{\mathrm{CD}}$. Eq. (1). It must be mentioned that the above equations are derived without requiring any decomposition of motions occurring in the system. This demonstrates that the recursion relation does not depend on the motions occurring in the system, as long as motional averaging happens, but rather on the tetrahedral geometry of the methylene group.

At this point of the development, it may be interesting to extend such calculation to cases where double bonds are present in lipid chains.

\section{B. Order tensor for unsaturated chains}

We envisage here the case of the oleoyl chain of POPC where the double bond stands at position 9-10 (Fig. 2). The molecular frame has then to be slightly modified. ${ }^{40} z$ is along the $\mathrm{C}_{9}-\mathrm{C}_{10}$ bond; $x$ is perpendicular to the plane defined by the $C_{9}-D$ (or $C_{10}-D$ ) bond and $z_{r}$ and $y$ is perpendicular to $x$ and $z$ axes. Let us note that following this definition, $S_{10}^{\mathrm{CC}}$ $=S_{z z}$. Writing similar equations as in (4) and (5), one obtains

$$
S_{9}^{C D}=\frac{1}{4} S_{z z}+\frac{3}{4} S_{y y}-\frac{\sqrt{3}}{2} S_{y z},
$$

$$
S_{10}^{\mathrm{CD}}=\frac{1}{4} S_{z:}+\frac{3}{4} S_{y y}+\frac{\sqrt{3}}{2} S_{y z}
$$

It must be mentioned that, in the present case, we have not been able to derive a recursion relation similar to $\mathrm{Eq}$. (I). However, one can take advantage that the double bond is rigid, that is to say no rotation around the $\mathrm{C}_{9}-\mathrm{C}_{10}$ can occur, and note the following: (i) $C_{9}-D$ is parallel to the $C_{10}-C_{11}$ bond and (ii) $\mathrm{C}_{10}-\mathrm{D}$ is parallel to the $\mathrm{C}_{8}-\mathrm{C}_{9}$ bond. Then, one should get for the cis double bond in position 9-10,

$$
s_{9}^{C D}=s_{11}^{C C} \text { and } s_{10}^{C D}=s_{9}^{C C} \text {. }
$$

This can be generalized in the case of a cis or trans double bond at positions $\mathrm{C}_{p}-\mathrm{C}_{p+1}$,

$$
\begin{aligned}
& \text { cis } S_{p}^{\mathrm{CD}}=S_{p+2}^{\mathrm{CC}} \text { and } S_{p+1}^{\mathrm{CD}}=S_{p}^{\mathrm{CC}}, \\
& \text { trans } S_{p}^{\mathrm{CD}}=S_{p+1}^{\mathrm{CD}} \text { and } S_{p+2}^{\mathrm{CC}}=S_{p}^{\mathrm{CC}} \text {, }
\end{aligned}
$$

where the notation for order parameters is similar to that indicated in Fig. 1. As will be discussed later, these properties may be of great interest for the assignment of labeled positions and allow calculation of $S^{\mathrm{CC}}$ order parameters for all carbon positions when going towards the polar head from the methyl terminal.

\section{The thermodynamical model}

In order to check the validity of Eq. (1) and analyze the $S^{\mathrm{CC}}$ profile, we calculated $S^{\mathrm{CC}}$ and $S^{\mathrm{CD}}$ by making use of a thermodynamical model. In what follows, we will briefly remind the general procedure employed to determine the energy of the chain. For more details, one may consult the following references. $34,41.4$ Under the assumption of fixed bond lengths $\left(l_{\mathrm{C} \cdot \mathrm{C}}=1.53 \AA\right)$ and bond angles $\left(\mathrm{C}_{k-1}-\mathrm{C}_{k}-\mathrm{C}_{k+1}=112^{\circ}\right.$ and $\left.\mathrm{H}-\mathrm{C}-\mathrm{H}=109^{\circ}\right),{ }^{42.43}$ the intemal dynamics of the chain is described in the frame of the rotational isomeric state (RIS) approximation. The rotation around the C-C bonds is assumed to be subject to a transgauche torsional potential. ${ }^{42,44,45}$ A stable configuration of the chain is then identified by a sequence of trans and gauche \pm . The energy of such a configuration is given in terms of intra- and intermolecular interactions. The intramolecular contribution arises from the isomerizations (rotation around the C-C bonds) and is assumed to be 0 for the trans state and $V_{g}$ for gauche \pm . For a given configuration, the intermolecular interaction is described by a mean field potential, ${ }^{34,35} V=-\epsilon \sum_{k=1}^{15} S_{z z}^{k}$, where $\epsilon$ gives the strength of the contribution. Let us note that steric criteria as detailed as in the original work ${ }^{4}$ yield 37227 allowed configurations for the $s n-1$ chain of DPPC. $S^{\mathrm{CC}}$ and $S^{\mathrm{CD}}$ order parameters may then be determined as

$$
S_{k}=\sum_{J} P_{J} S_{k, J}
$$

where $P_{J}$ and $S_{k, J}$ are respectively the fractional population (according to the Boltzman equation) and the order parameter of the position $k$ for the $J$ th configuration. ${ }^{41.46}$ 


\section{RESULTS AND DISCUSSION}

The $S^{\mathrm{CC}}$ order parameter formalism is of great interest for our understanding of conformational properties of acyl chains in lipid membranes. It affords calculation of conformer probabilities, acyl chain length and in combination with neutron scattering experiments, provides information about protrusion of lipids in the membrane. ${ }^{32}$ The basis of this formalism is a recursion relationship between $S^{\mathrm{CC}}$ and $S^{\mathrm{CD}}$ which has been derived by making use of Wigner matrices. ${ }^{20}$ This requires a complete decomposition of the different acyl chain motions and a set of Eulter angles allowing coordinate transformations from the initial tensor axis system to the laboratory frame of reference. We thus accounted for the isomerization around C-C bonds, lipid rotation around its long molecular axis and wobbling (oscillation around the bilayer normal). ${ }^{15,17}$ However, it was not clear whether the recursion relation depends or not on the dynamics occurring in the system. When using the concept of order tensor equations are derived without requiring any decomposition of motions and the average of order parameters is performed over time and space. Please note also that the introduction of the bilayer normal in the calculation could have been replaced by the magnetic field vector. Using this approach. we have thus shown that the recursion relation between $S^{\mathrm{CD}}$ and $S^{\mathrm{CC}}$ still stands and then does not depend on the type of motions of the acyl chain. It is rather a consequence of the tetrahedral geometry of the methylene group. Even if we have considered that both dihedral angles $\mathrm{H}-\mathrm{C}_{k}-\mathrm{H}$ and $\mathrm{C}_{k+1}-\mathrm{C}_{k}-\mathrm{C}_{k-1}$ are equal it will be shown in the following, from a calculation performed with the thermodynamical model where this condition is not rigorously satisfied, that the recursion relation still stands.

Let us now give a general comment conceming the determination of $S^{\mathrm{CD}}$ from molecular dynamics simulation. This is a powerful tool allowing theoretical calculation of physical properties of lipids embedded in a membrane. However, the time used when performing a molecular dynamics simulation (ps to ns time scale) could be too short for acyl chains to explore all their possible configurations (isomerization). As a consequence, due to insufficient time and space averaging, both deuterons on a methylene could be nonequivalent. It must be mentioned that usually in the literature, ${ }^{10} \mathrm{Eq}$. (3) (i.e., $S^{\mathrm{CD}}$ vs $S_{x x}$ and $S_{y y}$ ) is employed to calculate order parameters. We would like to stress that such an equation is only valid under the assumption that the order parameters on a methylene unit are equal. Care should be taken and the general definition of order parameters, Eq. (2), should preferentially be used, since the angle between the C-D bond and the bilayer normal can always be determined from the calculation. Note that it must also be possible to calculate the off-diagonal term $S_{x y}$, which could help as a criterion of convergence for the simulation since $S^{\mathrm{CD}}$ are often experimentally equivalent.

We shall now present the results concerning the calculation of $S^{\mathrm{CD}}$ and $S^{\mathrm{CC}}$ from the thermodynamical model. As mentioned above, the allowed configurations of the DPPC $s n-1$ chain are computationally generated. A given configuration is a sequence of gauche and trans from carbon $\mathrm{C}_{2}$ to the methyl terminal where the energy is calculated as de-
TABLE I. $S_{k}^{\mathrm{CD}}$ and $S_{k}^{\mathrm{CC}}$ order parameters as a function of carbon position. $k$. for the $s n-1$ chain of DPPC at $50^{\circ} \mathrm{C}$.

\begin{tabular}{|c|c|c|c|c|}
\hline$k$ & $S_{k}^{C D m}$ & $s_{k}^{\mathrm{COO}}$ & $2 S_{k}^{\mathrm{CDC}}$ & $S_{k}^{\mathrm{CCd}}+S_{k+1}^{\mathrm{CC}}$ \\
\hline 2 & -0.282 & n.d.e & 0.564 & n.d. \\
\hline 3 & -0.266 & 0.214 & 0.532 & 0.533 \\
\hline 4 & -0.289 & 0.319 & 0.578 & 0.579 \\
\hline 5 & -0.284 & 0.260 & 0.568 & 0.568 \\
\hline 6 & -0.286 & 0.308 & 0.572 & 0.571 \\
\hline 7 & -0.274 & 0.263 & 0.548 & 0.547 \\
\hline 8 & -0.269 & 0.284 & 0.538 & 0.537 \\
\hline 9 & -0.256 & 0.253 & 0.512 & 0.513 \\
\hline 10 & -0.249 & 0.260 & 0.498 & 0.498 \\
\hline 11 & -0.236 & 0.238 & 0.472 & 0.471 \\
\hline 12 & -0.226 & 0.233 & 0.452 & 0.452 \\
\hline 13 & -0.207 & 0.219 & 0.414 & 0.415 \\
\hline 14 & -0.190 & 0.196 & 0.380 & 0.380 \\
\hline 15 & -0.143 & 0.184 & 0.286 & 0.287 \\
\hline 16 & a.d.e & 0.103 & n.d.e & a.d. \\
\hline
\end{tabular}

From Ref. 4.

'From the thermodynamical model (vide supra).

"Calculated from column a.

Calculated from column b. The quantity $s_{k}^{c c}+s_{k+1}^{c c}$ is reported at line $k$ for ease of comparison with $-2 S_{k}^{\mathrm{CD}}$

Tot determined.

scribed above with the following parameters: $V g$ $=500 \mathrm{cal} / \mathrm{mol}$ and $\epsilon=0.3 \mathrm{kT} .{ }^{4,35-42}$ The order parameters have been calculated according to Eq. (14) and reported in Table I (columns $a$ and $b$ ). These values should be scaled by a molecular order parameter $\left(S_{\text {mol }}\right)$ accounting for the wobbling of the chain ${ }^{17}$ to be compot to experimental data. ${ }^{15,47}$

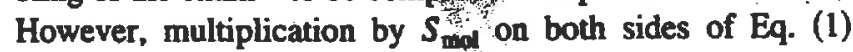
does not modify the recursion relation and the test can be performed at this level. From columns $a$ and $b$ we calculated the quantities $-2 S_{k}^{\mathrm{CD}}$ and $S_{k}^{\mathrm{CC}}+S_{k+1}^{\mathrm{CC}}$ (Table 1, columns c and d). For a given position $k$, data presented in these columns are identical within only an error of 0.001 . This is not surprising since the recursion relationship is a consequence of bond geometry. However, it is very interesting to note that Eq. (1) was established using a geometrical model ${ }^{20}$ based on a pure tetrahedral geometry, i.e., both $\mathrm{C}_{k-1}-\mathrm{C}_{k}-\mathrm{C}_{k+1}$ and $\mathrm{H}-\mathrm{C}-\mathrm{H}$ angles were taken to be $109.5^{\circ}$, whereas calculation from the thermodynamical model was performed with an angle of $112^{\circ}$ for $C_{k-1}-C_{k}-C_{k+1}$. Since the agreement between both methods is very good, this shows that the recursion relation does not depend too much (within a few degrees) on the dihedral condition.

We shall now comment the $S^{\mathrm{CD}}$ and $S^{\mathrm{CC}}$ profiles. Data from Table I have been plotted in Fig. 3 as a function of carbon position, $k$. $S^{\mathrm{CD}}$ exhibit a classical behavior, e.g., a plateau region followed by a decrease when going towards the methyl end ${ }^{47} S^{\mathrm{CC}}$ experiences a marked odd-even effect, especially at the beginning of the chain, with $S_{\text {odd }}^{\mathrm{CC}}<S_{\text {even }}^{\mathrm{CC}}$. This effect has been previously observed in the case of several lipids. ${ }^{20,32}$ It may be interesting to mention here that if one plots separately odd and even CC order parameters (not shown), one gets two smoothed independent curves, which join at around position 11. Odd order parameters start to increase from position 3 to 7 and then slowly decrease. The behavior for $S_{\text {even }}$ is markedly different since it continuously 


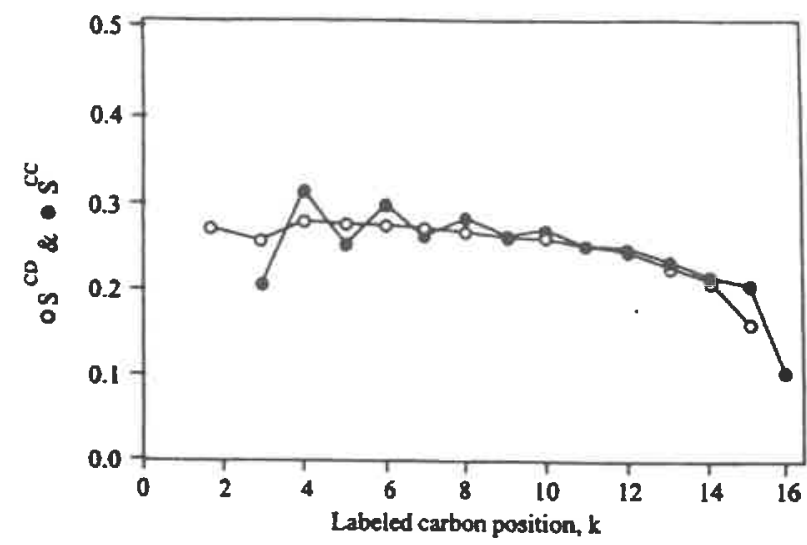

FIG. 3. $S_{k}^{C D}(O)$ and $S_{k}^{C C}(0)$ order parameters as a function of the carbon position, $k$, for the $s n-1$ chain of DPPC at $50^{\circ} \mathrm{C}$.

decreases from position 4 to the end. Let us make here a parallelism with the work of Lafleur. ${ }^{18}$ These authors described the $S^{\mathrm{CD}}$ order profile with a smoothed function, assuming a monotonic decrease of the order along the chain. In the same way, one could use two different empirical functions to approximate odd and even $S^{\mathrm{CC}}$ profiles. However, while monotonic decrease appears to be justified for $S_{\text {even }}$, the $S_{\text {odd }}$ profile should rather be described with a bell curve.

One can try to give an explanation to the odd-even effect by considering a chain in the all trans configuration with its long axis slightly tilted with respect to the rotational axis $Z_{\mathrm{D}}$ [Figs. 4(a) and 4(b)]. In such a case, all $S^{\mathrm{CD}}$ are equal since the angle between the $C-D$ bond and $Z_{D}$ remain the same with position $k$. On the contrary, $S^{C C}$ oscillates between two values. Indeed, the angle between the $C_{k}-C_{k+1}$ bond is similar to that of the $C_{k+2}-C_{k+3}$ bond (with respect to $Z_{D}$ ), whereas it differs from that of the $C_{k+1}-C_{k+2}$ bond. Let us remind here that in our computational calculations, the oddeven effect occurs eventhough no tilt of the acyl chain is accounted for. However, such a tilt of the chain can appear when a gauche defect stands on a methylene group [Fig. 4(c)]. Following the isomerization, the long axis of the rest of the chain is tilted with respect to $Z_{\mathrm{D}}$. This then would

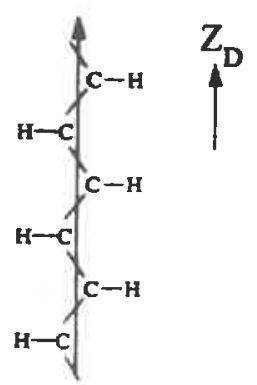

a)

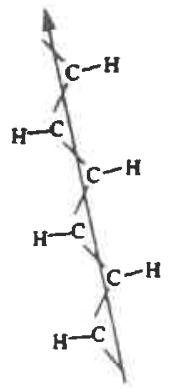

b)

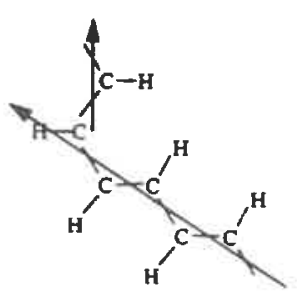

c)
FIG. 4. Schematic representation of an acyl chain needed for the description of the $S^{C C}$ add-even effect. $Z_{D}$ is the diffusion axis of the molecule, while the other arrows indicate the long axis of an acyl chain portion. (a) In the all trans case with the long axis parallel to $Z_{\mathrm{D}}$. (b) In the all trans case where the long axis is slightly tilted with respect to $Z_{D}$. (c) With an isomerization occurring in the middle of the chain: the long axis of the beginning of the chain is parallel to $Z_{D}$, while the rest is tilted. lead to odd-even $S^{\mathrm{CC}}$ for this part of the chain. In other words, the occurrence of the odd-even effect gives evidence that, in average, the chain is tilted with respect to $Z_{D}$. In our computational calculations this effect occurs at the beginning of the chain and slowly decreases on going to position 11 . This could mean that the average orientation of the chain is bent, the curvature decreasing as one reaches position 11 . Towards the chain end, the $S^{\mathrm{CC}}$ profile follows that of $S^{\mathrm{CD}}$ accounting for the increase in conformational freedom. The average axis orientation of this part of the chain would be parallel to $Z_{\mathrm{D}}$.

It must be reminded here that this odd-even effect has already been observed on $s n-2$ DL- DM- and DPPC, ${ }^{20}$ palmitic acid $^{21}$ and $s n-1$ DMPC chains. ${ }^{32}$ While the amplitude $(\Delta)$ of this odd-even effect was roughly constant as a function of temperature for palmitic acid and $s n-1$ chains, $s n-2$ chains experience a marked increase of $\Delta$ with temperature having started to roughly zero near the phase transition. This could mean that $s n-1$ chains are tilted, in average, independently of the temperature. At contrary and close to the phase transition, the average orientation of the beginning (from $\mathrm{C}_{3}$ ) of $s n-2$ chains would be parallel to the long molecular axis. This could be due to the fact that the carbonyl region of this chain is already bent. ${ }^{48-53}$ For higher temperature, the amplitude of the odd-even effect increases, in other words, the average orientation start to be bent what could be a consequence of the increase of lipid area. ${ }^{54}$

In order to reinforce the above remarks; it would be necessary to determine the orientational distribution function of the acyl chain. This could bexthe experimentally from IR-ATR experiments by determinigg the distribution function of the transition moment. ${ }^{55.56}$ This could also be studied from computational methods, with the method as described above, or from molecular dynamic simulations, where it is possible to calculate the average orientation of the chain and then predict whether it is tilted or not. These calculations are under progress in our laboratory.

We were not able to determine any recursion relationship between $S^{\mathrm{CD}}$ and $S^{\mathrm{CC}}$ in the case of unsaturated chains. When the double bond stands in position $\mathrm{C}_{9}-\mathrm{C}_{10}$, it is not possible, a priori, to calculate $S_{10}^{\mathrm{CC}}$ even if one knows $S_{11}^{\mathrm{CC}}$ and $S_{10}^{C D}$ (Fig. 2). However, we can use the advantage that the double bond is rigid for continuing to calculate $S^{\mathrm{CC}}$ order parameters along the whole chain. Let us first envisage the case of a cis double bond in position 9, 10 [Fig. 2(b)]. Let us suppose that it is possible, as we have shown above in the case of saturated chains, to calculate $S^{\mathrm{CC}}$ from the methyl terminus to $S_{11}^{C C}$, from a perdeuterated chain for instance. As a consequence of the double bond (no rotation occurring around the $\mathrm{C}_{9}-\mathrm{C}_{10}$ bond) $\mathrm{C}_{10}-\mathrm{C}_{11}$ and $\mathrm{C}_{9}-\mathrm{H}$ bonds on one hand and $\mathrm{C}_{9}-\mathrm{C}_{8}$ and $\mathrm{C}_{10}-\mathrm{H}$ bonds on the other hand remain parallel and then Eq. (11) applies. It is then possible continuing calculation of $S^{\mathrm{CC}}$ from $S_{9}^{\mathrm{CC}}$ to $S_{2}^{\mathrm{CC}}$. In the case of the trans conformation, a similar approach can be carried out from the experimental knowledge of all $S^{\mathrm{CD}}$ order parameters and especially $S_{9}^{\mathrm{CD}}$ and $S_{10}^{\mathrm{CD}}$. The latter order parameters can be predicted to be equal because the $\mathrm{C}_{9}-\mathrm{H}$ and $\mathrm{C}_{10} \mathrm{H}$ bonds are parallel [Fig. 2(a)]. Then for the same reasons [Eq. (13)] it comes: $S_{11}^{\mathrm{CC}}=S_{9}^{\mathrm{CC}}$. If one is able to cal- 
culate $S_{11}^{\mathrm{CC}}$ using Eq. (1) one can also determine $S_{9}^{\mathrm{CC}}$ and then all other $S^{\mathrm{CC}}$ order parameters on going towards the $\mathrm{C}_{2}$ position. One can therefore conclude that whatever the cis or trans conformation of the double bond, it is still possible to calculate $S^{\mathrm{CC}}$ for all positions, but that of the double bond, when going from the methyl-end towards the polar head using a perdeuterated sample and Eqs. (1), (12) and (13).

\section{CONCLUSION}

We have demonstrated from a thermodynamical point of view that there exists a recursion relationship linking $S^{\mathrm{CC}}$ to $S^{\mathrm{CD}}$ order parameters which offers a valuable basis to calculate physical parameters of saturated acyl chains embedded in biomembranes. This reinforces the geometrical model we already successfully applied to experimental data. ${ }^{20,21,32}$ However, it was unsuccessful, a priori, to develop an analog relation for unsaturated chains. Nonetheless, $s^{\mathrm{CC}}$ can still be calculated along the whole unsaturated chain except for one carbon of the double bond. We have also proposed an interpretation of the odd-even effect occurring on the $S^{\mathrm{CC}}$ order profile in terms of average tilt of the chain.

${ }^{i}$ H. L. Scott and S. Kalaskar, Biochemistry 28, 3687 (1989).

${ }^{2}$ M. J. Milik. J. Skolnick, and A. Kolinski. J. Phys. Chem. 96, 4015 (1992).

${ }^{3}$ T. Xiang, Biophys. J. 65.1108 (1993).

${ }^{4}$ A. Ferrarini, P. L. Nordio, G. J. More, R. H. Crepeau, and J. Freed, J. Chem. Phys. 89, 5707 (1989).

${ }^{5}$ E. T. Jaynes. Phys. Rev. 106, 620 (1957).

${ }^{6}$ D. Catalano. L. Di Bari, C. A. Veracini, G. N. Shilstone, and C. Zannoni, J. Chem. Phys. 94, 3928 (1990).

${ }^{7}$ R. W. Pastor, R. M. Venable, and M. Karplus, J. Chem. Phys. 89, 1112 (1988).

${ }^{8}$ P. van der Ploeg and H. J. C. Berendsen. J. Chem. Phys. 76, 3271 (1982).

${ }^{9}$ P. van der Ploeg and H. J. C. Berendsen, Mol. Phys. 49. 3271 (1983).

${ }^{10} \mathrm{H}$. Heller, M. Schaefer, and K. Schulten, J. Phys. Chem. 97, 8343 (1993).

" A. J. Robinson, W. G. Richards, P. J. Thomas, and M. M. Hann. Biophys. J. 68, 164 (1995).

12 J. Seelig, Q. Rev. Biophys. 10. 353 (1977).

${ }^{13}$ E. Olfield. M. Meadows, D. Rice, and R. Jacobs, Biochemistry 17, 2727 (1978).

14 J. Shen, N. A. Clark, P. S. Pershan, and E. B. Priestley, J. Chem. Phys. 66. $\$ 635$ (1977).

is J. H. Davis, Biochim. Biophys. Acta 737. 117 (1983).

${ }^{16}$ C. Morisson and M. Bloom, J. Chem. Phys. 101, 749 (1994).

${ }^{17}$ N. O. Peterson and S: Chan, Biochemistry 16, 2657 (1977).

${ }^{18}$ M. Lafleur, B. Fine, E. Sternin, P. R. Cuilis, and M. Bloom. Biophys. J. 56. 1037 (1989).

${ }^{19}$ C. R. Sanders and J. H. Prestegard, J. Am. Chem. Soc. 113, 1987 (19901)

${ }^{0} \mathrm{~J}$ J. P. Douliez. A. Léonard, and E. J. Dufourc, Biophys. J. 68, 1727 (1995)
${ }^{21}$ J. P. Douliez, B. Bechinger. J. H. Davis. and E. J. Dufourc, J. Phys. Chem. 100, 17083 (1996).

"2P. Meier, E. Ohmes, and G. Kothe. J. Chem. Phys. 85, 3598 (1986).

${ }^{23}$ C. Mayer. G. Gröbner. K. Muller, K. Weisz. and G. Kothe. Chem. Phys. Lett. 165. 155 (1988).

${ }^{2}$ E. J. Dufourc. C. Mayer, J. Stohrer, G. Althoff, and G. Kothe, Biophys. J. 61, 42 (1992).

${ }^{25}$ F. Auguste, P. Barois, L. Fredon, B. Clin. E. J. Dufourc. and A. M. Bellocq, J. Phys. II 4, 2197 (1994)

${ }^{36}$ M. Hong, K. Schmidt-Rohr, and D. Nanz. Biophys. J. 69. 1939 (1995).

${ }^{27}$ M. Hong, K. Schmidt-Rohr, and A. Pines, J. Am. Chem. Soc. 117, 3310 (1995).

${ }^{28}$ J. H. Gross, D. E. Warschawski, a1,d R. G. Griffin, J. Am. Chem. Soc. 119. 796 (1997).

${ }^{29}$ K. Hester, J. L. Ackerman. B. L. Neff, and J. S. Waugh, Phys. Rev. Lett. 36. 1081 (1976).

${ }^{30}$ D. Sandström, K. T. Summanen, and M. H. Levitt, J. Am. Chem. Soc. 116, 9357 (1994).

${ }^{3 i}$ K. Schmidt-Rohr, D. Nanz, L. Emsley, and A. Pines, J. Phys. Chem. 98, 6668 (1994).

${ }^{32}$ J. P. Douliez, A. Léonard, and E. J. Dufourc, J. Phys. Chem. 100, 18450 (1996).

${ }^{33}$ W. Maier and A. Saupe, Z. Naturforsch. A 13a, 564 (1958).

${ }^{34} \mathrm{~S}$. Marcelja, Biochim. Biophys. Acta 367, 164 (1974).

${ }^{35}$ J. W. Emsley, G. R. Luckhurst, and C. P. Stockley, Proc. R. Soc. London, Ser. A 381, 117 (1982).

${ }^{36} \mathrm{~S}$. Chandrasekhar, Liquid Crustals (Cambridge University Press, Cambridge, 1992).

${ }^{37}$ A. Saupe, Z. Naturforsch. A 19a, 161 (1964).

${ }^{38}$ P. G. De Gennes. The Physics of Liquid Crystals (Oxford University Press, London, 1974).

${ }^{39}$ E. Egberts and H. J. C. Berendsen, J. Chem. Phys. 89,3718 (1988).

${ }^{40} \mathrm{~J}$. Seelig and N. Waespe Sarcevic, Biochemistry 17, 3310 (1978).

${ }^{4} H$. Schindler and J. Seelig, Biochemistry 14, 2283 (1975).

42.P. J. Flory. Statistical Mechanics of Chify Molecules (Interscience, New York, 1969).

${ }^{43}$ R. A. Scott and H. A. Scheraga. J. Chem. Phys. 42, 2209 (1965); 44, 3054 (1966).

${ }^{44}$ P. B. Woller and E. W. Garbish, Jr., J. Am. Chem. Soc. 94, 5310 (1972).

${ }^{45}$ J. P. Rychaert and A. Bellemans, Chem. Phys. Lett. 30. 123 (1975).

${ }^{46}$ M. Karplus and J. N. Kushick, Macromolecules 14. 325 (1981).

${ }^{47}$ A. Seelig and J. Seelig, Biochemitry 13, 4839 (1974).

${ }^{18}$ H. Hauser. I. Pascher. and S. Sundell, Biochemistry 27, 9166 (1988).

${ }^{49}$ I. Pascher, M. Lundmark. P. G. Nyholm, and S. Sundell, Biochim. Biophys. Acta 1113. 339 (1992).

${ }^{50}$ B. A. Comell, Chern. Phys. Lett. 72, 462 (1980).

${ }^{51}$ A. Blume, W. Hübner, M. Müller, and H. D. Bäuerle, Ber. Bunsenges. Phys. Chem. 92, 964 (1988).

${ }^{52}$ W. Hübner and H. H. Mantsch, Biophys. J. 59. 1261 (1991).

${ }_{53}$ J. P. Douliez, Ph.D. thesis. University of Bordeaux, France, 1995.

${ }^{34}$ R. L. Thurmond, S. W. Dodd, and M. F. Brown, Biophys. J. 59, 108 (1991).

${ }^{55} \mathrm{C}$. Bolterauer and $\mathrm{H}$. Heller, Eur. Biophys. J. (in press).

${ }^{56} \mathrm{C}$. Bolterauer, Eur. Biophys. J. (in press). 